OSAHS

\title{
Evaluation of factors that influence the success rate of OSA treatment with a customised adjustable MAD device - a retrospective study
}

\author{
Analisi dei fattori che influenzano il successo della terapia delle OSA \\ con dispositivi MAD individualizzabili - studio retrospettivo
}

\begin{abstract}
Giuseppe Burlon¹, Michele Tepedino², Michele Laurenziello', Giuseppe Troiano1, Michele Cassano', Luigi Romano Raffaella Rinaldi ${ }^{4}$, Domenico Ciavarella ${ }^{1}$

${ }^{1}$ Department of Clinical and Experimental Medicine, University of Foggia, Italy; ${ }^{2}$ Department of Biotechnological and Applied Clinical Sciences, University of L'Aquila, Italy; ${ }^{3}$ Department of Otolaryngology Head and Neck Surgery, San Bassiano Hospital, Bassano del Grappa (VI), Italy; ${ }^{4}$ Department of Anatomical, Istological, Forensic and Locomotor System Sciences, Sapienza University of Rome, Italy
\end{abstract}

\section{SUMMARY}

The aim of the present study was to evaluate how the features of obstructive sleep apnoea (OSA) and the degree of mandibular advancement influence the outcomes of oral appliance therapy with a fully-customised mandibular advancement device (MAD) in an adult population. A total of 85 adult patients with mild to severe OSA were retrospectively selected. Polysomnography was taken before treatment and after 2 months treatment with overnight MAD. Treatment success was defined as a $>50 \%$ reduction in the Apnoea/Hypopnoea Index $(\mathrm{AHI})$ with a residual $\mathrm{AHI}<10$. Binary logistic regression was used to evaluate the effects of AHI, oxygen desaturation index (ODI), gender and age on the success rate of MAD therapy. MAD therapy was successful in $77.7 \%$ of patients, and the ODI was a significant predictor of treatment success. OSA treatment with the MAD was successful in reducing the AHI in adult patients. An ODI value smaller than 33.3 was a significant predictor of treatment success.

KEY WORDS: obstructive sleep apnoea, OSA, mandibular advancement, sleep parameters, oxygen desaturation index

\section{RIASSUNTO}

Lo scopo del presente lavoro è stato quello di valutare in che modo le caratteristiche della sindrome delle Apnee Ostruttive del Sonno (OSA) ed il grado di avanzamento mandibolare influenzano $i$ risultati della terapia con dispositivi di avanzamento mandibolare (MAD) individualizzabili in una popolazione adulta. Sono stati selezionati retrospettivamente 85 pazienti adulti con OSA da lieve a severa. È stata eseguita una polisonnografia pre-trattamento e dopo 2 mesi di terapia notturna con MAD. La terapia è stata definita di successo se ha comportato una riduzione dell'Indice di Apneal Ipopnea $(A H I)>50 \%$ con un AHI residuo $<10$. Una regressione logistica binaria è stata usata per valutare l'effetto di AHI, indice di desaturazione dell'ossigeno (ODI), sesso ed età sul tasso di successo della terapia con MAD. Tale trattamento ha avuto successo nel 77,7\% dei pazienti, e l'ODI è risultato essere un predittore significativo della probabilità di successo della terapia. Il trattamento delle OSA con il dispositivo MAD utilizzato si è rivelato efficace nel ridurre l'AHI nei pazienti adulti. Un valore di ODI inferiore a 33,3 è indicativo della possibilità di successo del trattamento.

PAROLE CHIAVE: apnea ostruttiva del sonno, OSA, avanzamento mandibolare, parametri del sonno, indice di desaturazione dell'ossigeno

\section{Introduction}

Obstructive sleep apnoea (OSA) is defined as a syndrome characterised by repeated collapse of the upper airways during sleep, resulting in airflow suspension that requires arousal to recover patency of the airways and
Received: May 13, 2019

Accepted: July 27, 2019

Published online: June 10, 2020

\section{Correspondence}

Michele Tepedino

V.le San Salvatore, Edificio Delta 6, 67100 L'Aquila, Italy

Tel. +39 0862434794. Fax +390862434782

E-mail:m.tepedino@hotmail.it

Funding

None.

Conflict of interest

Giuseppe Burlon is the owner of the patent and the registered trademarks for Occlusion ${ }^{\circledast}$, nonrusso+ ${ }^{\circledR}$, and Protrusor ${ }^{\circledR}$. All other Authors declare no conflict of interest.

How to cite this article: Burlon G, Tepedino M, Laurenziello M, et al. Evaluation of factors that influence the success rate of OSA treatment with a customised adjustable MAD device - a retrospective study. Acta Otorhinolaryngol Ital 2020;40:297-303. https://doi. org/10.14639/0392-100X-N0307

() Società Italiana di Otorinolaringoiatria e Chirurgia Cervico-Facciale

\section{cc) (1) $\ominus$}

This is an open access article distributed in accordance with the CC-BY-NC-ND (Creative Commons Attribution-NonCommercial-NoDerivatives 4.0 International) license. The article can be used by giving appropriate credit and mentioning the license, but only for non-commercial purposes and only in the original version. For further information: https:// creativecommons.org/licenses/by-nc-nd/4.0/deed.en 
normal breathing ${ }^{1}$. A diagnosis of OSA is confirmed after overnight polysomnography, when an Apnoea-Hypopnoea Index (AHI, number of apnoea/hypopnoea events per hour of sleep) greater than 5 and a complaint of daytime sleepiness are observed ${ }^{2}$. Hypoxaemia/hypercapnia derived from the reduced airflow, fragmented sleep caused by frequent arousals, exaggerated fluctuations in heart rhythm, blood pressure and intrathoracic pressure are responsible for severe sequelae like cardiovascular morbidity and mortality, neurocognitive impairment, systemic inflammation, increased risk of driving accidents and poorer quality of life ${ }^{3}$. It is a significant health-related problem that affects $5.9 \%$ of women and $12.5 \%$ of men in the general population over 40 years ${ }^{4}$. A study on an Italian population reported a prevalence of $2.7 \%$ in a range between 30 to 69 years of age ${ }^{5}$.

The treatment options for this kind of patients comprise behavioural changes (e.g., weight loss and reduced consumption of alcohol), sleep position training, continuous positive airway pressure (CPAP), mandibular advancement devices (MAD) and upper airway surgery. According to the Practice Parameters of Care of the American Academy of Sleep Medicine (AASM), CPAP is the gold standard for treatment of OSA in adult patients, particularly for moderate to severe cases ${ }^{6}$. CPAP therapy is currently provided by the Italian National Health Service, being introduced among the Essential Levels of Care since 2017. This welfare policy is based on a concept of health that has evolved considerably: it is no longer about being healthy and disease-free, but rather about thorough well-being from physical, psychological and social perspectives ${ }^{7}$. Thus, the Italian healthcare system guarantees potentially universal access to care, without any distinction of personal and social conditions. However, nonadherence to CPAP treatment is high ${ }^{8}$, and therefore MAD therapy is recommended by the AASM as a first-line treatment in mild to moderate OSA cases or as an alternative therapy for severe cases, non-compliant with $\mathrm{CPAP}^{6}$. A MAD acts by forcing the mandible to a forward position, thus increasing the upper airway's volume by widening the velopharynx, stabilising the soft palate and hyoid bone, stretching the tongue muscles and preventing the posterior rotation of the mandible ${ }^{9}$. Although CPAP is more effective in reducing the AHI, this difference is overcome by greater compliance and acceptance of MAD therapy ${ }^{10,11}$.

The results of a systematic review demonstrated that different types of MADs have different efficacy in terms of mandibular protrusion and reduction of OSA events ${ }^{12}$, with fully customisable devices showing superior performance ${ }^{10}$. While there is consensus on a comparable efficacy in AHI reduction between CPAP and MAD in mild to moderate OSA patients, there is still some uncertainty regarding severe OSA patients: while some authors reported promising results for severe OSA patients ${ }^{10}$, a systematic review of the relevant literature was unable to perform a subgroup analysis to evaluate the efficacy of MAD therapy related to OSA severity ${ }^{11}$. In addition, the current literature provides only a limited number of studies that describe the amount of mandibular advancement, considered as an important factor for both treatment outcomes and side effects ${ }^{13}$.

The aim of the present retrospective study was to evaluate how OSA features and the degree of mandibular advancement influence the outcomes of oral appliance therapy with a fully-customised MAD in an Italian adult population.

\section{Materials and methods}

This retrospective study was carried according to the Strengthening the Reporting of Observational Studies in Epidemiology (STROBE) guidelines.

The records of OSA patients treated at a University Dental Clinic, from January 2011 to January 2018, were screened for the following inclusion criteria:

- Epworth Sleepiness Scale > 10;

- diagnosis of OSA confirmed by an overnight polysomnography;

- results of drug-induced sleep endoscopy (DISE) confirming the positive effect of mandibular advancement on airway obstruction;

- overnight MAD therapy with a fully-customisable device (Protrusor ${ }^{\circledR}$, nonrusso+ ${ }^{\circledR}$, Dr Giuseppe Burlon, Belluno, Italy);

- age $>20$ years old;

- a follow-up of 2 months with polysomnographic evaluation;

- completeness of diagnostic records.

Exclusion criteria were: smoking habit, BMI greater than $34 \mathrm{~kg} / \mathrm{m}^{2}$, previous surgical treatment of the maxillofacial complex or the upper airway, presence of fixed oral appliance or mobile prosthetic rehabilitation, patients in treatment with CPAP or nCPAP, patients with severe cardiovascular disease, presence of temporomandibular joint disorder.

The procedures followed were in accordance with the Helsinki Declaration of 1975, as revised in 1983. The records were retrieved retrospectively, analysed anonymously and patients signed written informed consent to participate to future research at the time that the records were taken. The Protocol was approved by the Ethics Committee of the University of L'Aquila, Italy (protocol no. 9032). 
To detect a moderate to small effect size $(0.3)$ having a I type error of 0.05 and a power of $0.8,64$ subjects were needed ${ }^{13}$.

For all patients, data on $\mathrm{AHI}$ and the 3\% Oxygen Desaturation Index (3\% ODI, number of desaturation events greater than $3 \%$ of the baseline value per hour of sleep) were retrieved from pre-treatment (T0) polysomnography. According to AASM guidelines, patients with an AHI between 5 and 15 were defined as mild OSA, patients with an AHI ranging between 15 and 30 were defined as moderate OSA, while those with an AHI greater than 30 were considered to have severe OSA ${ }^{2}$. For mild to moderate OSA patients, MAD therapy was the first-choice of treatment, while severe OSA patients started MAD therapy after non-adherence to CPAP therapy.

\section{Instrumental evaluation}

Each patient received complete overnight polysomnography (PSG). All subjects were evaluated for one night in a sleep laboratory using a portable device, the Embletta system (Flaga, Reykjavik, Iceland). The recording was performed after one night of adaptation to the hospital setting. The airflow was monitored by a nasal cannula and by an oral thermistor. The thoracic-abdominal movements of all subjects were detected through two piezoelectric belts. Overnight continuous recordings of oxygen saturation were obtained by finger pulse oximetry. Snoring was recorded by a microphone placed at the neck, and note was taken of ECG findings and sleep position.

Apnoea was defined as the cessation of airflow lasting $10 \mathrm{sec}$; and hypopnoea was defined as a discrete reduction (two-thirds) of airflow and/or abdominal rib-cage movements lasting $10 \mathrm{sec}$ associated with a $>3 \%$ decrease in oxygen saturation. The number of events per hour was obtained by dividing the total number of events by the total sleep time (TST) and was defined as the AHI. The oxygen desaturation index (ODI) was also measured, as were the number of arterial oxyhemoglobin saturation dips $\geq 3 \%$. Nocturnal hypoxaemia was evaluated in terms of percentage of total sleep time with oxyhaemoglobin saturation $<90 \%$. Sleep apnoea was defined using AHI $\geq 10$ per hour. The test was repeated with oral appliance three months later in all patients.

Patient enrolled in the present study had previously had a DISE to evaluate the collapse area. In the operating room, electrocardiography, pulse oximetry, non-invasive blood pressure and bispectral index (BIS) were monitored. Propofol was used during DISE. Propofol $2 \mathrm{mg} / \mathrm{kg}$ was intravenously administrated at DISE start; $1 \mathrm{mg} / \mathrm{kg}$ was given for maintenance. The maximum dosage of propofol was $3 \mathrm{mg} / \mathrm{kg}$. A flexible fiberoptic endoscope (Type ENF-
GP, Olympus Europe GmbH, Hamburg, Germany) was introduced by one experienced ENT surgeon in the awake patient to evaluate the awake upper airway state.

When target sedation was reached, a soft mandibular protrusion was produced by use of a protrusor appliance.

\section{The device}

The MAD used for the present study is a customisable and adjustable device. It is composed of two resin splints connected by two threaded titanium bars with two titanium screw each, which constitutes the protrusive component of the device (Figs. 1, 2). The head of the titanium screws allows for lateral movements of the mandible, while keeping the mandible in a forward and lower position. The titanium bars have laser marks every $2 \mathrm{~mm}$ to facilitate the titration of the degree of mandibular advancement.

\section{Treatment protocol}

All patients were instructed to wear the MAD: an initial mandibular advancement of $70 \%$ of the total possible

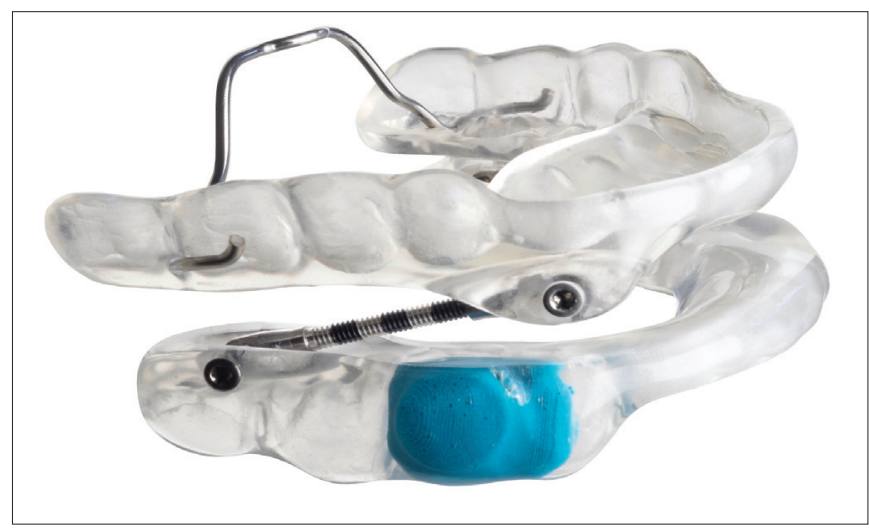

Figure 1. The Protrusor ${ }^{\circledR}$ MAD device. It is made of two resin splints connected by two threaded titanium bars, which can be regulated to reach the desired amount of mandibular advancement. A chip for compliance monitoring is embedded in the lower resin splint.

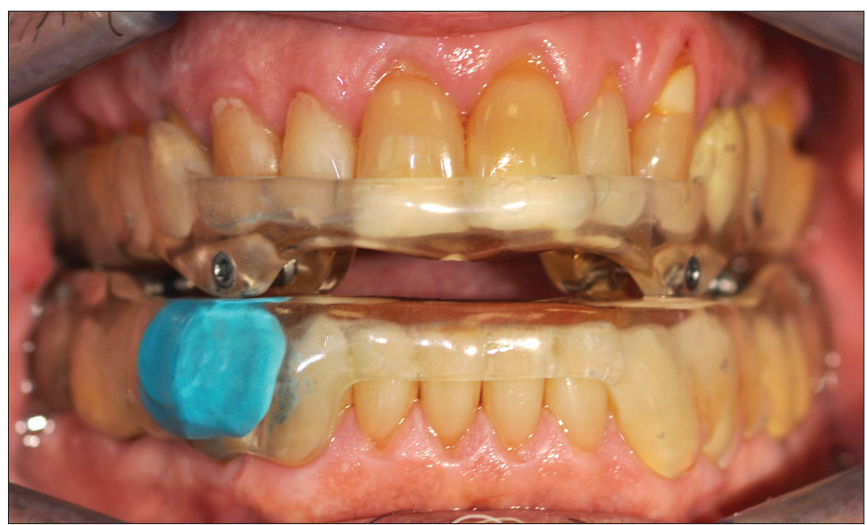

Figure 2. The Protrusor ${ }^{\circledR}$ MAD device in place after individual titration. 
protrusive movement was set using and intraoral gauge (Occlusion ${ }^{\circledR}$, nonrusso $+{ }^{\circledR}$, Dr Giuseppe Burlon, Belluno, Italy) (Fig. 3). During the following visits every 15 days, the therapeutic mandibular protrusion was adjusted on an individual basis with 1 or $2 \mathrm{~mm}$ increments, until the most comfortable position that allowed improvement of symptoms was reached: this position was established based on the symptoms reported by patients and their bed partner. The amount of the final mandibular advancement was recorded for each patient as a percentage of the maximal protrusive movement achievable.

Post-treatment (T1) AHI and 3\% ODI values were retrieved from another polysomnography after a period of 2 months of treatment.

The therapy was considered successful based on the residual AHI value: patients showing $\geq 50 \%$ AHI reduction compared to baseline and a $\mathrm{T} 1 \mathrm{AHI} \leq 10$ were considered good responders. On the other hand, patients showing a reduction $<50 \%$ of $\mathrm{AHI}$, but with a residual $\mathrm{AHI}>10$ were considered bad responders and unsuccessful cases.

\section{Statistical analysis}

For AHI and 3\% ODI values, the T1-T0 difference was also calculated and reported both as a raw value and a percentage of the T0 value. Descriptive statistics and overall success rate were calculated. A Shapiro-Wilk normality test was used to evaluate the distribution of all variables. Binary logistic regression was performed to study the effects of age, gender, AHI value at T0, ODI $3 \%$ at T0, and mandibular advancement on being classified as a good or a bad responder. The predictors were included in the model with a forward stepwise method. The first type error was set as $\mathrm{P}<0.05$.

\section{Results}

Complete records for 85 patients were available out of the 150 initially screened; the remaining incomplete diagnostic records of the other subjects were excluded from further study. The study sample was composed of 72 males (mean age $55.3 \pm 12.3$ ) and 13 females (mean age $65.5 \pm 7.9$ ); other demographic characteristics are reported in Table I. Based on the T0 AHI value, 26 patients were classified as mild OSA, 36 patients as moderate OSA, and 23 patients as severe OSA.

Descriptive statistics are reported in Table I. According to the criteria reported in the Materials and methods, 66 patients were considered good responders and 19 patients poor responders. Therefore, the treatment was considered successful in $77.7 \%$ of patients. All patients showed good compliance with MAD therapy. Some of

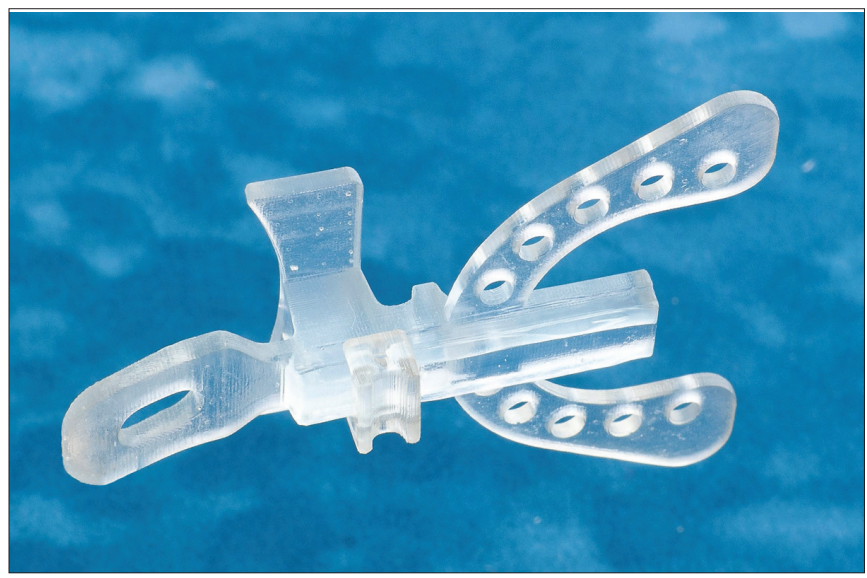

Figure 3. The Occlusion ${ }^{\circledR}$ device used to register the ideal mandibular protrusion, and to transfer the desired mandibular position to the dental laboratory for manufacturing the MAD device.

the severe OSA patients who initially refused CPAP, but whose MAD therapy was unsuccessful, returned to CPAP therapy after the period considered for the present study. No side effects on the temporo-mandibular joint (TMJ) were reported by any patient.

The logistic model was able to correctly predict $81.2 \%$ of cases. The regression model included only the variables ODI $3 \%$ at T0 and the Constant (Tab. II), whereas the other predictors were not significant and were excluded by the forward stepwise method (Tab. III). The odds ratio for the ODI $3 \%$ variable was 0.92 , meaning that a 1 -point decrease of ODI multiplies the odds of being a good responder by 0.92 . The calculated critical value for ODI $3 \%$ was ODI $<$ intercept/coefficient $<3.36 / 0.09<33.3$. Therefore, an ODI value smaller than 33.3 was needed to be classified as a good responder.

\section{Discussion}

MADs are a valuable treatment modality for patients with OSA: several reports have confirmed they are associated with improvement in the quality of life, daytime sleepiness, cardiac autonomic function ${ }^{14}$, and functional and cognitive outcomes that are comparable to CPAP therapy ${ }^{11}$. The results of the present study showed that MAD therapy with the customised adjustable device studied can successfully treat $77.7 \%$ of adult patients. Comparing our data with the existing literature is not simple because there is large heterogeneity in the parameters used to consider a treatment successful or not ${ }^{3}$. Some authors reported a success (defined as post-treatment $\mathrm{AHI}<10$ ) rate of $72 \%$ when using a customised non-adjustable MAD ${ }^{15}$. Other authors observed clinical success (defined as post-treatment AHI < 10) in $54 \%$ of adult patients ${ }^{16}$. Fernandez-Julian et al. reported 
Table I. Descriptive statistics for good responders and bad responders.

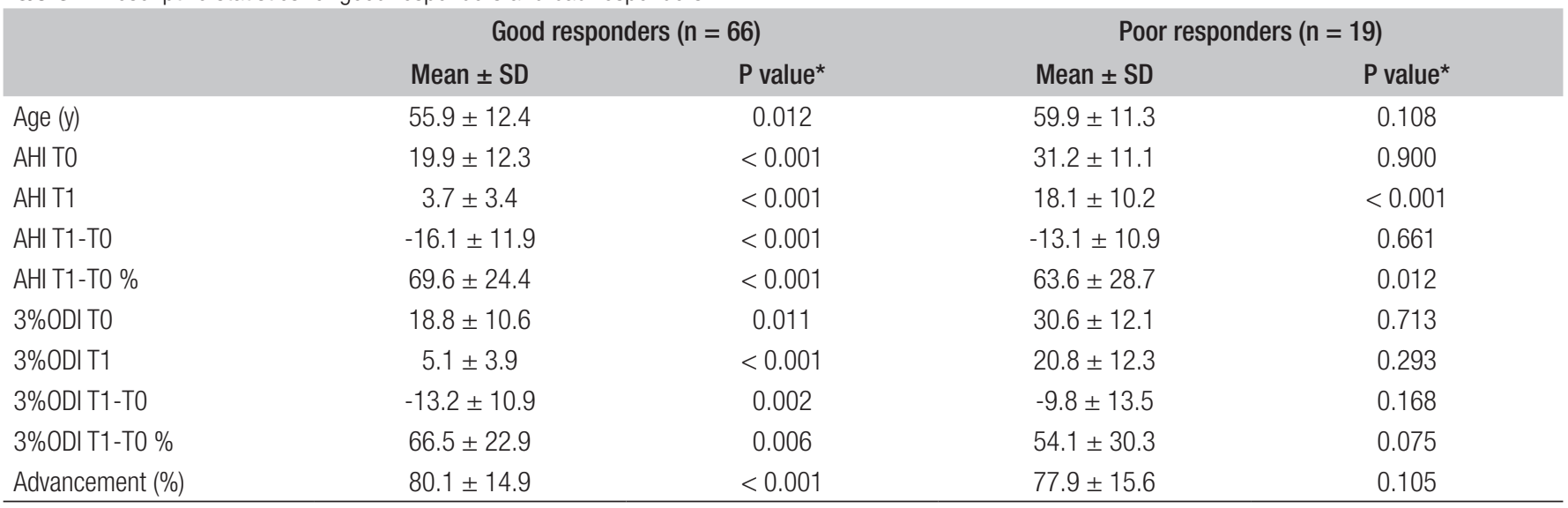

*: $P$ value from the Shapiro-Wilk normality test.

Table II. Stepwise binary logistic regression $(n=85)$.

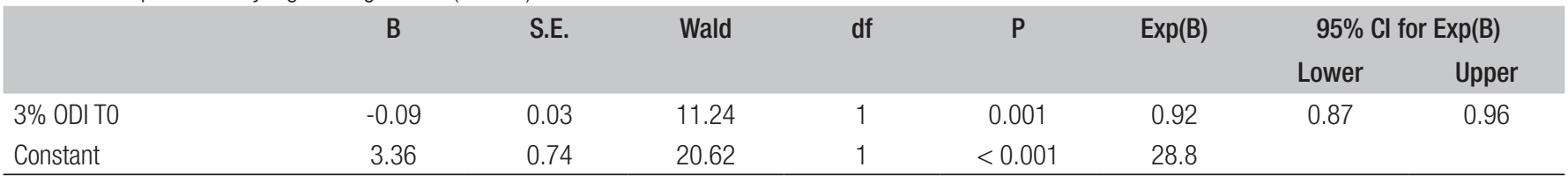

Table III. Variables excluded from the binary logistic regression model $(\mathrm{n}=85)$.

\begin{tabular}{lccc} 
& Score & Df & P value \\
Age & 3.079 & 1 & 0.079 \\
Gender & 0.085 & 1 & 0.770 \\
AHI TO & 0.155 & 1 & 0.694 \\
Advancement \% & 1.384 & 1 & 0.239 \\
Overall statistics & 4.935 & 4 & 0.294 \\
\hline
\end{tabular}

successful $(\mathrm{AHI}<10$ and Epworth Sleepiness Score $<10)$ treatment in $50 \%$ of adult patients with moderate to severe $\mathrm{OSA}^{3}$. Mints et al. showed a higher success (defined as post-treatment $\mathrm{AHI}<10$ ) rate of $80 \%$ using a customised adjustable appliance in a sample of 510 patients comprising mild, moderate and severe OSA ${ }^{10}$, and concluded that treatment success is not dependent on the baseline degree of severity of OSA. This finding is in contrast with our results: a significant $(\mathrm{P}=0.001)$ effect of baseline 3\% ODI value on treatment success was found (Tab. II). Higher values of 3\% ODI predicted an unsuccessful treatment outcome, with a calculated threshold value of 33.3 oxygen desaturation events per hour of sleep. The 3\% ODI value was a stronger predictor than the AHI value, the latter being excluded from the regression model due to collinearity (0.792) with the $3 \%$ ODI variable.

These results appear to confirm current guidelines suggesting MAD treatment as the first choice therapy for mild to moderate OSA patients, and as an alternative to CPAP for severe OSA ${ }^{11}$. The main advantage of MADs, even in severe OSA patients, is its significantly higher compliance and self-reported usage compared to CPAP ${ }^{11}$. On the other hand, OSA treatment with MAD is also associated with some side effects. In the short term, muscular and articular tenderness, gingival irritation, mouth dryness and excessive salivation can occur: these side effects can be easily managed and even prevented ${ }^{17}$. Regarding TMJ pain, it has been reported in the literature in $12.5 \%$ of patients, and usually manifests in the early phases of treatment ${ }^{18}$. A study on FEM models revealed that the TMJ does not experience significant stress, regardless of the amount of mandibular advancement, suggesting that the TMJ discomfort referred by patients could be due to altered muscle dynamics, since the forward posturing of the mandible increases muscular activity ${ }^{19,20}$.

On the other hand, long-term side effects are more complex and involve skeletal and dental modifications. The mechanism behind these changes is the same as those described for orthodontic functional appliances: when the muscles are stretched by the device due to the forward posturing of the mandible, forces are transmitted to the bones and the teeth, and the long duration of these forces produces changes in skeletal morphology and teeth position ${ }^{21}$. The dental and skeletal changes are progressive, and thus patients should be monitored over time, and are also dependent on the duration of the MAD therapy ${ }^{13}$. In the present study, no relevant short-term side-effects were reported, while long-term effects could not be evaluated. Regarding the amount of mandibular advancement, the 
present study demonstrated that this is not a parameter that influences clinical outcomes of therapy (Tab. III). On the contrary, some authors reported that greater mandibular advancements were more effective in improving polysomnographic parameters ${ }^{22,23}$. However, Lamont et al. observed that for some OSA patients, excessive mandibular advancement causes an increase in airway obstruction ${ }^{24}$. Other authors recommended MAD treatment with an advancement of no more than $50 \%$ of the maximum protrusive movement, because greater advancements did not produce further improvements in AHI reduction ${ }^{25}$. The results of the present study seem to confirm that titration of mandibular advancement through subsequent steps is important, because each patient has their own therapeutic mandibular position that should be recognised, a position that does not necessarily coincide with the maximum achievable protrusion ${ }^{12}$.

Other factors like the patient's age and gender were not significant predictors of the success of MAD treatment, confirming the findings of other studies ${ }^{3}$.

The main limitation of the present study is its retrospective nature. We took care to avoid any selection bias, and all patients with incomplete records were excluded. Another limitation is the short follow-up, which precludes us from making statements on the long-term success of MAD therapy with the proposed device. Possible future improvements of the present protocol could be evaluation of other patient related parameters such as body mass and neck circumference ${ }^{26}$, and assessment of quality of life, since other parameters in addition to AHI can explain the clinical success of therapy ${ }^{3,27}$. Further studies could also take into account skeletal features ${ }^{28,29}$ and volumetric assessment of the upper airway ${ }^{30,31}$.

\section{Conclusions}

The MAD studied was effective in reducing the AHI value below 10 in $77.7 \%$ of patients in a population of adults with mild to severe OSA. The main predictor of the success of MAD treatment was a 3\% ODI value below 33.3.

\section{References}

1 Pillar G, Shehadeh N. Abdominal fat and sleep apnea: the chicken or the egg? Diabetes Care 2008;31(Suppl 2):2008. https://doi. org/10.2337/dc08-0715

2 Berry R, Brooks R, Gamaldo C, et al. The AASM Manual for the scoring of sleep and associated events: rules, terminology and technical specifications. Version 2.2 Darien, IL: American Academy of Sleep Medicine; 2015. https://aasm.org/resources/pdf/scoring-manual-preface.pdf

3 Fernández-Julián E, Pérez-Carbonell T, Marco R, et al. Impact of an oral appliance on obstructive sleep apnea severity, quality of life, and biomarkers. Laryngoscope 2018;128:1720-6. https://doi.org/10.1002/lary.26913
4 Heinzer R, Marti-Soler H, Haba-Rubio J. Prevalence of sleep apnoea syndrome in the middle to old age general population. Lancet Respir Med 2016;4:e5-6. https://doi.org/10.1016/S2213-2600(16)00006-0

5 Cirignotta F, D'Alessandro R, Partinen M, et al. Prevalence of every night snoring and obstructive sleep apnoeas among 30-69-year-old men in Bologna, Italy. Acta Neurol Scand 1989;79:366-72. https:// doi.org/10.1111/j.1600-0404.1989.tb03802.x

6 Kushida CA, Morgenthaler TI, Littner MR, et al. Practice parameters for the treatment of snoring and Obstructive Sleep Apnea with oral appliances: an update for 2005. Sleep 2006;29:240-3. https://doi. org/10.1093/sleep/29.2.240

7 Rinaldi R. Health in the $21^{\text {st }}$ Century: new rights come to the fore? Clin Ter 2018;169:e149-50. https://doi.org/10.7417/T.2018.2070

8 Weaver TE, Grunstein RR. Adherence to continuous positive airway pressure therapy: the challenge to effective treatment. Proc Am Thorac Soc 2008;5:173-8. https://doi.org/10.1513/pats.200708-119MG

9 Chan ASL, Sutherland K, Schwab RJ, et al. The effect of mandibular advancement on upper airway structure in obstructive sleep apnoea. Thorax 2010;65:726-32. https://doi.org/10.1136/thx.2009.131094

10 Mintz SS, Kovacs R. The use of oral appliances in obstructive sleep apnea: a retrospective cohort study spanning 14 years of private practice experience. Sleep Breath 2018;22:541-6. https://doi.org/10.1007/ s11325-018-1643-5

11 Schwartz M, Acosta L, Hung YL, et al. Effects of CPAP and mandibular advancement device treatment in obstructive sleep apnea patients: a systematic review and meta-analysis. Sleep Breath 2018;22:555-68. https://doi.org/10.1007/s11325-017-1590-6

12 Ahrens A, McGrath C, Hägg U. A systematic review of the efficacy of oral appliance design in the management of obstructive sleep apnoea. Eur J Orthod 2011;33:318-24. https://doi.org/10.1093/ejo/cjq079

13 Bartolucci ML, Bortolotti F, Martina S, et al. Dental and skeletal longterm side effects of mandibular advancement devices in obstructive sleep apnea patients: a systematic review with meta-regression analysis. Eur J Orthod 2019;41:89-100. https://doi.org/10.1093/ejo/cjy036

14 Glos M, Penzel T, Schoebel C, et al. Comparison of effects of OSA treatment by MAD and by CPAP on cardiac autonomic function during daytime. Sleep Breath 2016;20:635-46. https://doi.org/10.1007/ s11325-015-1265-0

15 Marklund M, Stenlund H, Franklin KA. Mandibular advancement devices in 630 men and women with obstructive sleep apnea and snoring: tolerability and predictors of treatment success. Chest 2004;125:1270-8. https://doi.org/10.1378/chest.125.4.1270

16 Yoshida K. Effects of a mandibular advancement device for the treatment of sleep apnea syndrome and snoring on respiratory function and sleep quality. Cranio 2000;18:98-105. https://doi.org/10.1080/08 869634.2000.11746120

17 de Almeida FR, Lowe AA, Tsuiki S, et al. Long-term compliance and side effects of oral appliances used for the treatment of snoring and obstructive sleep apnea syndrome. J Clin Sleep Med 2005;1:143-52.

18 Cistulli PA, Gotsopoulos H, Marklund M, et al. Treatment of snoring and obstructive sleep apnea with mandibular repositioning appliances. Sleep Med Rev 2004;8:443-57. https://doi.org/10.1016/j. smrv.2004.04.002

19 Heidsieck DSP, Koolstra JH, de Ruiter MHT, et al. Biomechanical effects of a mandibular advancement device on the temporomandibular joint. J Cranio-Maxillofacial Surg 2018;46:288-92. https://doi. org/10.1016/j.jcms.2017.11.015

20 Di Palma E, Tepedino M, Chimenti C, et al. Effects of the functional orthopaedic therapy on masticatory muscles activity. J Clin Exp Dent 2017;9:e886-91. https://doi.org/10.4317/jced.53986

21 Kinzinger GSM, Lisson JA, Frye L, et al. A retrospective cephalometric investigation of two fixed functional orthodontic appliances 
in class II treatment: functional mandibular advancer vs. herbst appliance. Clin Oral Investig 2018;22:293-304. https://doi.org/10.1007/ s00784-017-2111-5

22 de Almeida FR, Bittencourt LR, de Almeida CIR, et al. Effects of mandibular posture on obstructive sleep apnea severity and the temporomandibular joint in patients fitted with an oral appliance. Sleep 2002;25:507-13.

23 Marklund M, Franklin KA, Sahlin C, et al. The effect of a mandibular advancement device on apneas and sleep in patients with obstructive sleep apnea. Chest 1998;113:707-13. https://doi.org/10.1378/ chest.113.3.707

24 Lamont J, Baldwin DR, Hay KD, et al. Effect of two types of mandibular advancement splints on snoring and obstructive sleep apnoea. Eur J Orthod 1998;20:293-97. https://doi.org/10.1093/ejo/20.3.293

25 Tegelberg A, Walker-Engström M-L, Vestling O, et al. Two different degrees of mandibular advancement with a dental appliance in treatment of patients with mild to moderate obstructive sleep apnea. Acta Odontol Scand 2003;61:356-62. https://doi. org/10.1080/00016350310007130

26 Ciavarella D, Tepedino M, Chimenti C, et al. Correlation between body mass index and obstructive sleep apnea severity indexes - a retrospective study. Am J Otolaryngol 2018;39:388-91. https://doi. org/10.1016/j.amjoto.2018.03.026

27 Romandini M, Gioco G, Perfetti G, et al. The association between periodontitis and sleep duration. J Clin Periodontol 2017;44:490-501. https://doi.org/10.1111/jcpe.12713

28 Guarda-Nardini L, Manfredini D, Mion M, et al. Anatomically based outcome predictors of treatment for obstructive sleep apnea with intraoral splint devices: a systematic review of cephalometric studies. J Clin Sleep Med 2015;11:1327-34. https://doi.org/10.5664/jcsm.5198

29 Stipa C, Cameli M, Sorrenti G, et al. Relationship between cephalometric parameters and the apnoea-hypopnoea index in OSA patients: a retrospective cohort study. Eur J Orthod 2020;42:101-6. https://doi. org/10.1093/ejo/cjz038

30 Di Carlo G, Fernandez Gurani S, Pinholt EM, et al. A new simple three-dimensional method to characterize upper airway in orthognathic surgery patient. Dentomaxillofac Radiol 2017;46:20170042. doi:10.1259/dmfr.20170042

31 Luzzi V, Di Carlo G, Saccucci M, et al. Craniofacial morphology and airflow in children with primary snoring. Eur Rev Med Pharmacol Sci 2016;20:3965-71. 